\title{
ORIENTAÇÕES DE ALTA HOSPITALAR PARA O DESEMPENHO DO AUTOCUIDADO APÓS A CIRURGIA CARDÍACA: REVISÃO INTEGRATIVA
}

\author{
HOSPITAL DISCHARGE GUIDELINES FOR \\ SELF-CARE PERFORMANCE AFTER CARDIAC \\ SURGERY: INTEGRATIVE REVIEW
}

\section{ORIENTACIONES DE ALTA HOSPITALARIA PARA EL RENDIMIENTO DEL AUTOCUIDADO DESPUÉS DE LA CIRUGÍA CARDÍACA: REVISIÓN INTEGRATIVA}

\author{
Thaina Laurentino Santos ${ }^{1}$ \\ Manoela Gomes Grossi Laprano ${ }^{2}$ \\ Ana Paula da Conceição ${ }^{3}$
}

\begin{abstract}
Como citar este artigo: Santos TL, Laprano MGG, Conceição AP. Orientações de alta hospitalar para O desempenho do autocuidado após a cirurgia cardíaca: revisão integrativa. Rev baiana enferm. 2020;34:e-35284.

Objetivo: identificar as orientações de saúde necessárias, para que o paciente em pós-operatório de cirurgia cardíaca possa desempenhar comportamentos de autocuidado após a alta hospitalar. Método: revisão integrativa, seguindo as etapas propostas por Whittemore, limitada aos idiomas português, inglês e espanhol, e publicação entre 2007 e 2018. Utilizaram-se as bases de dados disponíveis nas bibliotecas virtuais PubMed e Biblioteca Virtual em Saúde e o instrumento de Ursi para extração dos dados. Classificaram-se os artigos conforme o nível de evidência. Resultados: foram incluídos seis artigos. Os achados possibilitaram a criação de nove categorias que agrupam as orientações de saúde para o desempenho do autocuidado após alta hospitalar decorrente de cirurgia cardíaca. Conclusão: atividades de vida diária, alimentação, atividade e exercício físico, sintomas psicológicos, controle de fatores de risco, complicações, terapia medicamentosa, manejo dos sintomas e pele foram orientações de saúde identificadas para o desempenho do autocuidado após alta hospitalar de pacientes submetidos a cirurgia cardíaca.
\end{abstract}

Descritores: Revascularização Miocárdica. Procedimentos Cirúrgicos Cardiovasculares. Valvas Cardíacas. Cuidados Pós-Operatórios. Educação de Pacientes como Assunto. Autocuidado.

Objective: to identify the necessary health guidelines, so that the patient in the postoperative period of cardiac surgery can perform self-care behaviors after hospital discharge. Method: integrative review, following the steps proposed by Whittemore, limited to the Portuguese, English and Spanish languages, and publication between 2007 and 2018. The databases used were available on the virtual libraries PubMed and Virtual Health Library and the Ursi instrument for data extraction. The articles were classified according to the level of evidence. Results: six articles were included. The findings allowed creating nine categories that group the health guidelines for self-care performance after hospital discharge from cardiac surgery. Conclusion: activities of daily living, diet, physical activity and exercise, psychological

\footnotetext{
Enfermeira. Especialista em enfermagem em cardiologia. Residente do Programa de Residência em Enfermagem Cardiovascular do Instituto Dante Pazzanese de Cardiologia. São Paulo, São Paulo, Brasil. thainalaurentino95@gmail.com. https://orcid.org/0000-0002-2286-6791.

Enfermeira. Mestre em Ciências. Coordenadora do Programa de Residência em Enfermagem Cardiovascular e do Núcleo de Enfermagem do Programa de Residência Multiprofissional em Saúde Cardiovascular no Instituto Dante Pazzanese de Cardiologia. São Paulo, São Paulo, Brasil. https://orcid.org/0000-0002-5237-0754.

Enfermeira. Mestre em Enfermagem. Chefe da Unidade de Internação Adulto II no Instituto Dante Pazzanese de Cardiologia e Interlocutora da Sistematização da Assistência de Enfermagem na Secretaria de Estado de Saúde do Estado de São Paulo. São Paulo, São Paulo, Brasil. https://orcid.org/0000-0002-I598-807X.
} 
symptoms, control of risk factors, complications, drug therapy, management of symptoms and skin were health guidelines identified for the performance of self-care after hospital discharge of patients undergoing cardiac surgery.

Descriptors: Myocardial Revascularization. Cardiovascular Surgical Procedures. Heart valves. Post-Operative Care. Patient Education as Topic. Self Care.

Objetivo: identificar las orientaciones de salud necesarias, para que el paciente en el período postoperatorio de la cirugía cardiaca pueda realizar comportamientos de cuidado personal después del alta hospitalaria. Método: revisión integradora, siguiendo los pasos propuestos por Whittemore, limitada a los idiomas portugués, inglés y español, y publicación entre 2007 y 2018. Fueron utilizadas las bases de datos disponibles en las bibliotecas virtuales PubMed y Biblioteca Virtual en Salud y el instrumento Ursi para la extracción de datos. Los artículos fueron clasificados de acuerdo con el nivel de evidencia. Resultados: se incluyeron seis artículos. Los hallazgos permitieron la creación de nueve categorias que agrupan las orientaciones de salud para el desempeño del autocuidado después del alta hospitalaria debido a la cirugía cardiaca. Conclusión: las actividades de la vida diaria, la dieta, la actividad $y$ el ejercicio físico, los síntomas psicológicos, el control de los factores de riesgo, las complicaciones, la terapia farmacológica, el manejo de los sintomas y la piel fueron orientaciones de salud identificadas para el desempeño del autocuidado después del alta hospitalaria de los pacientes sometidos a cirugía cardíaca.

Descriptores: Revascularización Miocárdica. Procedimientos Quirúrgicos Cardiovasculares. Válvulas Cardiacas. Cuidados Postoperatorios. Educación del Paciente como Asunto. Autocuidado.

\section{Introdução}

As Doenças Cardiovasculares (DCV) são responsáveis por 31\% de todas as mortes em âmbito global, constituindo-se na principal causa de morte nos países em desenvolvimento ${ }^{(1)}$. No cenário nacional, elas ocupam um papel de destaque, respondendo por $27,7 \%$ dos óbitos ${ }^{(2)}$. As Cirurgias Cardiovasculares (CVS) fazem parte dos tratamentos previstos em diretrizes e consensos para as DCV, sendo as mais comuns a Cirurgia de Revascularização do Miocárdio (CRM) e a correção de doenças valvares ${ }^{(3)}$.

Devido ao elevado grau de complexidade, as cirurgias cardiovasculares apresentam altas taxas de readmissão hospitalar ${ }^{(4)}$. As complicações pós-cirúrgicas mais prevalentes, como as cardíacas, neurológicas e infecciosas, podem fazer parte do período pós-operatório ${ }^{(5)}$. Desse modo, a transição dos cuidados do paciente torna-se uma prática essencial, que exige planejamento e educação em saúde para garantir a assistência à saúde no domicílio ${ }^{(6-7)}$.

Observa-se ainda ausência de sistemática no planejamento da educação para a alta hospitalar, baixa qualidade das orientações prestadas e relatos de pacientes com dúvidas acerca de quais cuidados e como desempenhá-los ${ }^{(8)}$. A falta de organização do preparo para alta gera impacto na qualidade de vida dos doentes e de seus cuidadores, pois se trata de um momento crítico de transição e adaptação de suas atividades de vida diárias ${ }^{(9-10)}$, impostas pelo procedimento cirúrgico e futuro tratamento clínico de prevenção de eventos cardiovasculares.

Nesse contexto, o enfermeiro, como membro da equipe de saúde, atua na coordenação e execução dos cuidados da transição do hospital para o domicílio. Presta orientações efetivas, que favorecem o uso das medicações e o gerenciamento do autocuidado, aumentando a adesão ao tratamento e reduzindo a taxa de reinternação hospitalar ${ }^{(6)}$.

O processo de transição pode ser facilitado ou dificultado por circunstâncias pessoais, condições ambientais, sociais ${ }^{(11)}$, pela idade, complexidade clínica do paciente, cognição, alfabetização e presença de jargões nas instruções ${ }^{(12-13)}$. O planejamento educativo para a transição dos cuidados deve incluir a avaliação das necessidades de aprendizagem dos doentes e seus familiares ${ }^{(11)}$, identificando suas limitações e dúvidas sobre como controlar a dor, a náusea, a constipação e os problemas emocionais, que, dentre muitos outros, podem permear a fase pós-operatória. O cuidado individualizado centrado no paciente pode ajudar a aumentar a confiança, fortalecendo o paciente e promovendo o autocuidado $^{(4)}$. 
Desse modo, define-se o autocuidado como um processo de escolha baseado em experiências pessoais e sociais, realizado pelo indivíduo, a fim de alcançar uma estabilidade ou melhora de sinais e sintomas diante de um quadro de agravo de sua saúde. Alguns fatores, como alfabetização de saúde, estado psicossocial, cognição, nível de escolaridade e grau da doença, podem associar-se à não adesão ao autocuidado ${ }^{(14)}$. O empoderamento do indivíduo sobre sua condição de saúde é de suma importância para obtenção de sua autonomia, sendo o modo mais adequado para tomada de decisões pertinentes, resultando na manutenção da qualidade de vida e prevenção de hospitalizações recorrentes ${ }^{(15)}$.

Estratégias eficazes, para que os pacientes consigam realizar o autogerenciamento dos sinais e sintomas no pós-operatório, são imprescindíveis para a recuperação ${ }^{(16)}$. Essas, por sua vez, devem ter um caráter emancipador e considerar o paciente de forma holística, integrando sua comunidade e sua família, a fim de que haja troca de experiências e não somente imposição de informações sobre condições de saúde-doença ${ }^{(17)}$.

O enfermeiro empoderado por meio de evidências científicas que subsidiam a sua prática clínica é um profissional capacitado para o desenvolvimento dessas estratégias educativas em saúde de modo eficaz, a fim de motivar o autocuidado do indivíduo ${ }^{(18-19)}$.

Entretanto, as diretrizes que norteiam a assistência ao paciente submetido a $\mathrm{CRM}^{(20)}$, correção de doenças valvares ${ }^{(21)}$ e que apresentam Angina Instável (AI) e Infarto Agudo do Miocárdio (IAM) ${ }^{(22)}$, não trazem, de forma abrangente, os conteúdos necessários ao ensino do paciente para o desempenho do autocuidado após a sua alta hospitalar.

Nesse contexto, compreendendo o papel do enfermeiro na transição do cuidado, o presente estudo tem como objetivo realizar uma revisão integrativa para identificar as orientações de saúde necessárias, para que o paciente em pós-operatório de cirurgia cardíaca possa desempenhar comportamentos de autocuidado, após a alta hospitalar.

\section{Método}

Realizou-se um estudo de revisão integrativa sobre as orientações de saúde necessárias para o desempenho do autocuidado após a alta hospitalar de pacientes submetidos a cirurgia cardíaca. Para a presente revisão, seguiram-se as etapas: identificação do problema, pesquisa bibliográfica, avaliação dos dados, análise dos dados e apresentação da revisão de literatura ${ }^{(23)}$.

A pergunta de pesquisa foi definida com base na estratégia PICO, em que P (paciente) são os pacientes adultos em pós-operatório de cirurgia cardíaca de revascularização, válvula e cirurgia combinada; I (intervenção) representa estudos primários que abordem as orientações necessárias ao paciente ou educação/ensino para o desempenho dos cuidados após a alta hospitalar; e O (resultado) são as medidas de autocuidado/aprendizado do paciente. Nesta revisão integrativa, o C (controle), terceiro elemento, não foi utilizado. A questão norteadora do estudo foi: Quais orientações de saúde os pacientes em pós-operatório de cirurgia cardíaca precisam receber para desempenharem o autocuidado após sua alta hospitalar?

Para a busca dos artigos, utilizaram-se as bases de dados disponíveis nas bibliotecas PubMed e Biblioteca Virtual em Saúde (BVS). A estratégia de busca baseou-se nos Descritores em Ciências da Saúde da Bireme (DeCS) e no Medical Subject Headings (MeSH), além dos operadores booleanos AND e OR com os termos: revascularização miocárdica, procedimentos cirúrgicos cardiovasculares, valvas cardíacas, cuidados pós-operatórios, educação de pacientes como assunto, e autocuidado.

A busca foi limitada aos idiomas português, inglês e espanhol, disponíveis na íntegra, e publicados no período de 2007 a 2018. Os resultados desta revisão foram comparados com as diretrizes $^{(20-22)}$ de manejo do paciente coronariano e valvar. Devido a isso, o recorte temporal a partir de 2007 foi definido conforme a data de publicação dessas normas, pois estudos anteriores a essa data poderiam abordar orientações discordantes das diretrizes. A busca de artigos 
ocorreu por meio de acesso on-line às bases de dados, nos meses de janeiro a março de 2019.

Para atender aos critérios de inclusão, os artigos deveriam responder aos elementos definidos na estratégia PICO, estarem disponíveis na íntegra e dentro do recorte temporal. Artigos de todos os tipos de revisão foram considerados critério de exclusão. Para a seleção dos estudos foi realizada inicialmente a leitura de títulos e resumos. Na sequência, fez-se a revisão por pares das publicações na íntegra, sendo selecionadas as que obedeceram aos critérios de elegibilidade.

A extração dos dados dos estudos incluídos na revisão foi realizada por dois revisores, de forma independente, mediante aplicação de instrumento que permitiu organizar os achados, facilitando a comparação dos estudos e a visualização dos dados $^{(24)}$. Posteriormente, as orientações foram discriminadas, com base na questão norteadora do estudo, e categorizadas em linhas de cuidados.
Os artigos foram classificados conforme $\mathrm{O}$ nível de evidência, de acordo com a seguinte classificação: (I) revisões sistemáticas ou metanálises de relevantes ensaios clínicos; (II) ensaio clínico randomizado; (III) ensaio clínico sem randomização; (IV) estudos de coorte e de caso-controle; (V) revisão sistemática de estudos descritivos e qualitativos; (VI) um único estudo descritivo ou qualitativo; (VII) opinião de um comitê de especialistas ${ }^{(25)}$.

As estratégias de buscas às bases de dados retornaram 193 estudos cujos títulos e resumos foram analisados independentemente. Desses, 54 estavam duplicados, 56 eram estudos de revisão, 1 não atendia o critério de idioma (chinês) e 25 não abordavam o período pós-operatório. Ao todo, 57 estudos foram lidos na íntegra, sendo excluídos 51, pois 24 não descreviam as orientações realizadas e 27 não abordavam o autocuidado. Assim, foram incluídos na revisão 6 artigos (Fluxograma 1).

Fluxograma 1 - Processo de seleção dos estudos primários

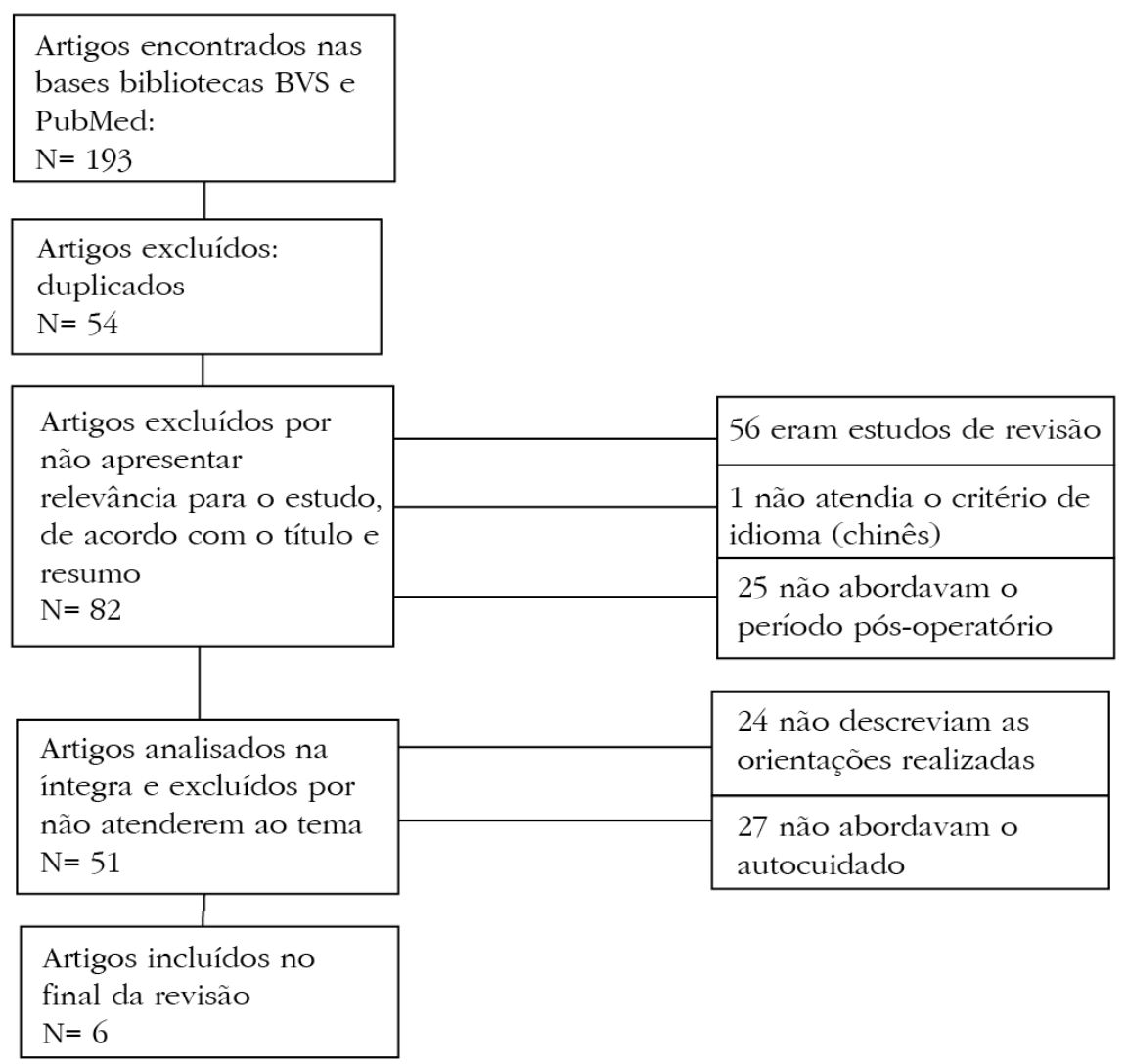

Fonte: Elaboração própria. 
A síntese dos achados é apresentada em formato de quadro. A análise se fez com base nas categorias estabelecidas, conforme apresentado a seguir.

\section{Resultados}

Compuseram a amostra final seis artigos, lidos na íntegra por pares. Em relação às bases de dados, cinco estudos foram localizados tanto na PubMed quanto na BVS, todos indexados na
Medline. Um estudo encontrava-se na BVS, indexado no Lilacs. Cinco artigos estão no idioma inglês e um em português. Observando-se a força das evidências obtidas nos artigos, encontraram-se três (50\%) artigos com nível de evidência II e dois (16,66\%) com nível III e VI, cada. Um estudo não foi classificado, pois não se enquadrou no referencial metodológico adotado neste estudo. A síntese dos estudos identificados na revisão é apresentada no Quadro 1.

Quadro 1 - Caracterização dos estudos nas bases de dados consultadas, segundo autores, título, ano de publicação, tipo de estudo e classificação de acordo com nível de evidência

\begin{tabular}{|c|c|c|c|c|}
\hline Autores & Título & Ano & Tipo de estudo & $\begin{array}{c}\text { Nivel de } \\
\text { Evidência }\end{array}$ \\
\hline $\begin{array}{l}\text { Yildiz T, } \\
\text { Gürkan S, } \\
\text { Gür Ö, Ünsal C, } \\
\text { Göktaş SB, } \\
\text { Özen Y }^{(26)}\end{array}$ & $\begin{array}{l}\text { Effect of standard versus patient- } \\
\text { targeted in-patient education on } \\
\text { patients' anxiety about self-care after } \\
\text { discharge from cardiovascular surgery } \\
\text { clinics }\end{array}$ & 2014 & $\begin{array}{l}\text { Coorte } \\
\text { prospectivo }\end{array}$ & II \\
\hline Fredericks $S^{(27)}$ & $\begin{array}{l}\text { The influence of country of origin on } \\
\text { engagement in self-care behaviours } \\
\text { following heart surgery: a descriptive } \\
\text { correlational study }\end{array}$ & 2012 & $\begin{array}{l}\text { Não } \\
\text { experimental } \\
\text { descritivo }\end{array}$ & VI \\
\hline Fredericks $S^{(28)}$ & $\begin{array}{l}\text { Timing for delivering individualized } \\
\text { patient education intervention to } \\
\text { Coronary Artery Bypass Graft patients: } \\
\text { An RCT }\end{array}$ & 2009 & $\begin{array}{l}\text { Ensaio clínico } \\
\text { randomizado }\end{array}$ & II \\
\hline $\begin{array}{l}\text { Cebeci } \mathrm{F}_{3} \\
\text { Çelik SS }^{(29)}\end{array}$ & $\begin{array}{l}\text { Discharge training and counselling } \\
\text { increase self-care ability and reduce } \\
\text { postdischarge problems in CABG } \\
\text { patients }\end{array}$ & 2008 & $\begin{array}{l}\text { Estudo } \\
\text { prospectivo e } \\
\text { quase- } \\
\text {-experimental }\end{array}$ & III \\
\hline $\begin{array}{l}\text { Fredericks S, } \\
\text { Sidani S, } \\
\text { Shugurensky D }\end{array}$ & $\begin{array}{l}\text { The effect of anxiety on learning } \\
\text { outcomes post-CABG }\end{array}$ & 2008 & $\begin{array}{l}\text { Ensaio clínico } \\
\text { randomizado }\end{array}$ & II \\
\hline $\begin{array}{l}\text { Gentil LLS, } \\
\text { Silva RM, } \\
\text { Benavente SBT, } \\
\text { Costa ALS } \\
\end{array}$ & $\begin{array}{l}\text { Manual educativo de cuidados no } \\
\text { pós-operatório de revascularização } \\
\text { miocárdica: uma ferramenta para } \\
\text { pacientes e familiares }\end{array}$ & 2017 & $\begin{array}{l}\text { Pesquisa } \\
\text { metodológica }\end{array}$ & - \\
\hline
\end{tabular}

Fonte: Elaboração própria.

A Tabela 1 apresenta as orientações de saúde oferecidas aos pacientes em pós-operatório de cirurgia cardíaca, identificadas nos estudos, para o desempenho do autocuidado após alta hospitalar, segundo a classificação por categorias, expostas pelo número de seu referencial. 
Tabela 1 - Orientações de autocuidado após alta hospitalar de pacientes em pós-operatório de cirurgia cardíaca, por número de estudos e Referencial. São Paulo, SP, Brasil - jan-mar 2019. (N=6) (continua)

\begin{tabular}{l} 
Orientações de autocuidado \\
\hline Atividades de Vida Diária \\
Alterações no padrão de sono e repouso \\
Higiene corporal (tomar banho, vestir-se) \\
Atividade sexual \\
Atividades sociais (frequentar locais públicos)
\end{tabular}

Número de estudos $\%$ (n)

$66,6(4)$

33,3 (2)

33,3 (2)

16,6 (1)

$83,3(5)$

66,6 (4)

66,6 (4)

33,3 (2)

$66,6(4)$

66,6 (4)

33,3 (2)

$83,3(5)$

33,3 (2)

16,6 (1)

66,6 (4)

33,3 (2)

33,3 (2)

33,3 (2)

16,6 (1)

$66,6(4)$

49,9 (3)

49,9 (3)

33,3 (3)

33,3 (2)

16,6 (1)

$83,3(5)$

33,3 (2)

$16,6(1)$

16,6 (1)

$83,3(5)$

49,9 (3)

49,9 (3)

33,3 (2)
Referencial

27-28,30-31

26,31

26,29

31

26-28,30-31

26-27,29,31

27-28,30-31

28,30

26,29-31

27-28,30-31

29,31

26-29,31

28,31

31

26,28,30-31

26,31

26,31

29,31

26

27-28,30-31

27-28,30

26,29,31

28,30-31

29,31

29

27-31

26,31

31

31

26-28,30-31

27-28,30

27-28,30

28,30 
Tabela 1 - Orientações de autocuidado após alta hospitalar de pacientes em pós-operatório de cirurgia cardíaca, por número de estudos e Referencial. São Paulo, SP, Brasil - jan-mar 2019. (N=6) (conclusão)

\begin{tabular}{l|c|c}
\hline Orientações de autocuidado & $\begin{array}{c}\text { Número de estudos } \\
\mathbf{\%}(\mathbf{n})\end{array}$ & Referencial \\
\hline Pele & $66,6(4)$ & $26,29-31$ \\
$\quad$ Cuidados com as incisões cirúrgicas & $66,6(4)$ & $27-28,30-31$ \\
Complicações da ferida operatória & $33,3(2)$ & 28,30 \\
Dor nas incisões & $33,3(1)$ & 31 \\
Cicatrização da ferida operatória & &
\end{tabular}

Fonte: Elaboração própria.

Não foram identificados estudos publicados nos últimos dez anos que tratassem de orientações para o pós-operatório de cirurgias de correção valvar.

Em relação ao contexto em que as orientações para o desempenho do autocuidado foram realizadas nos estudos, observou-se: os temas foram abordados durante a aplicação de ações educativas junto aos pacientes ${ }^{(26-27,29,30)}$, ou serviram de base para a construção de ferramentas de aprendizagem para a educação do paciente em pós-operatório de $\mathrm{CRM}^{(28)}$, ou, ainda, foram utilizadas para compor instrumentos de avaliação de comportamentos de autocuidado ${ }^{(31)}$.

O ensino das orientações para o desempenho do autocuidado após a alta hospitalar foi realizado por meio de estratégias, como ligação telefônica ${ }^{(26-27)}$, vídeo ${ }^{(26)}$ e manual educativo ${ }^{(29-30)}$, a maior parte realizada na fase pós-operatória. Cinco estudos ${ }^{(26-30)}$ realizaram uma avaliação prévia das necessidades individuais de aprendizagem dos pacientes em relação às atividades de autocuidado. Dois estudos ${ }^{(26-27)}$ evidenciaram maior desempenho de comportamentos de autocuidado após as intervenções educativas e um $^{(30)}$ relatou impacto positivo na capacidade de autocuidado dos pacientes e no alívio dos problemas encontrados no período pós-operatório.

Um estudo ${ }^{(29)}$ mostrou que a educação individualizada diminui os níveis de ansiedade sobre o autocuidado após a alta, e outro ${ }^{(31)}$ evidenciou que o país de origem influencia a quantidade de comportamentos de autocuidado que os indivíduos realizaram após a cirurgia cardíaca.
Observou-se em um estudo que o momento da intervenção educativa antes ou após alta não influencia no desempenho do autocuidado ${ }^{(26)}$. Um estudo relatou que os níveis de ansiedade têm potencial negativo na retenção do conhecimento, no autocuidado e na experiência dos sintomas durante o pós-operatório ${ }^{(27)}$.

\section{Discussão}

A alta hospitalar é uma etapa do cuidado organizada antecipadamente. O processo de educação envolve paciente e familiar, compartilhando informações de maneira efetiva, com diferentes recursos de comunicação, e certificando-se de que as dúvidas e os problemas foram esclarecidos e solucionados ${ }^{(18,32)}$.

Tendo em vista que, na presente revisão de literatura, nenhum dos estudos que compuseram a amostra possui nível I e apenas a metade possui nível II de evidência, percebe-se a necessidade de pesquisas que produzam resultados com fortes níveis de evidência científica, a fim de subsidiar o ensino do paciente para o desempenho do autocuidado após a alta hospitalar.

Embora nenhum dos 38 tópicos de orientação tenha atingido $100 \%$ de concordância entre os artigos, todos abordavam no mínimo um tópico dentro de cada categoria. As orientações de saúde mais citadas para os pacientes em pós-operatório de cirurgia cardíaca (5-83,3\%) foram administração de medicamentos ${ }^{(27-31)}$, reações emocionais ${ }^{(26-29,31)}$, constipação ${ }^{(26-28,30-31)} \mathrm{e}$ $\operatorname{dor}^{(26-28,30-31)}$. 
De fato, a administração de medicamentos é uma das orientações mais fornecidas pelos enfermeiros durante a internação e na alta hospitalar. Apesar disso, a não aderência medicamentosa em doentes com condições crônicas é expressiva, variando entre 50\% e 80\%. Assim, nas orientações sobre os medicamentos, é necessário, além de reforçar a importância da adesão ao tratamento, considerar a particularidade medicamentosa, prevenindo interações entre os fármacos. Além disso, é preciso identificar as dificuldades de cada paciente, como a condição financeira e a dificuldade na identificação dos medicamentos, fatores que impactam diretamente na aderência medicamentosa ${ }^{(6,33-34)}$. Apesar desses dados, as orientações sobre a aquisição e interação medicamentosa foram citadas por apenas um artigo.

Os níveis de ansiedade e depressão aumentam no período pós-operatório de cirurgia cardíaca. Evidenciam-se relatos de medo e incerteza em relação ao futuro. As reações emocionais iniciam ainda no pré-operatório, aumentando o uso de analgésicos, os riscos de morbidade pós-cirúrgica e o tempo de internação hospitalar. Portanto, os sintomas psicológicos devem ser avaliados de forma rotineira antes mesmo da cirurgia, e fazer parte do planejamento de alta hospitalar, para minimizar o impacto que podem trazer após o procedimento ${ }^{(35)}$.

Um dos diagnósticos de enfermagem mais prevalentes no pós-operatório é o risco de constipação. Isso ocorre, pois o período pós-operatório é marcado por mudanças na rotina do paciente, que impactam na alimentação e na limitação das atividades físicas, resultando em motilidade gastrointestinal diminuída. Assim, é um tema que deve fazer parte das orientações para transição dos cuidados no domicílio $^{(36)}$.

A dor é um sintoma prevalente e considerado um dos principais estressores percebidos pelos pacientes no pós-operatório de cirurgia cardíaca. Está associada à piora da independência funcional e à recuperação cirúrgica retardada. Seu adequado manejo pode contribuir para reduzir o tempo de internação hospitalar e diminuir taxas de complicação no período pós-operatório ${ }^{(37-40)}$.
Também frequentes nos artigos - 4 casos, correspondendo a $66,7 \%$ - foram as orientações sobre cuidados com as incisões cirúrgicas, complicações pós-operatórias (sintomas de cansaço, fadiga e dispneia) ${ }^{(27-28,30-31)}$, consultas ambulatoriais de acompanhamento ${ }^{(26,28,30-31)}$, retorno às atividades habituais ${ }^{(27-28,30-31)}$, exercício físico $^{(26,29-31)}$, restrição de líquidos ${ }^{(27-28,30-31)}$, dieta especial $^{(26-27,29,31)}$ e alterações no padrão de sono e repouso $^{(27-28,30-31)}$.

As principais diretrizes de cuidado aos pacientes portadores de coronariopatias e valvulopatias abordam diversas orientações identificadas neste estudo. Tais orientações referem-se à atividade sexual, dieta, tabagismo, terapia medicamentosa, reações emocionais, depressão, prevenção de fatores de risco cardiovasculares, atividades habituais, exercício físico e cuidados com a ferida operatória ${ }^{(20-22)}$.

Embora as diretrizes direcionem tópicos para orientação e transição dos cuidados para o domicílio, não abrangem temas importantes para empoderar os pacientes na adoção de comportamentos de autocuidado no pós-operatório de cirurgia cardíaca.

Verificou-se que as orientações a respeito de atividades sociais (frequentar locais públicos) ${ }^{(31)}$, depressão ${ }^{(31)}$, uso de drogas ilícitas ${ }^{(26)}$, hipertermia e febre ${ }^{(29)}$, aquisição e interação de medicamentos $^{(31)}$ foram abordados em apenas um $\operatorname{artigo~(18,4\% ).~Apesar~disso,~estudo~}{ }^{(41)}$ refere que os aspectos físicos, psicológicos, interpessoais e sociais da saúde são inseparáveis, sendo papel da enfermagem ajudar o paciente a entendê-los.

A necessidade de mudanças no estilo de vida após cirurgia cardíaca pode levar o paciente a evitar ou recusar a participação em eventos sociais, acarretando isolamento social, principalmente no gênero masculino ${ }^{(42)}$. Como o isolamento social e a solidão impactam no aumento dos índices de mortalidade, devido aos efeitos sobre a saúde mental e as doenças cardiovasculares $^{(43)}$, esses temas devem fazer parte das orientações para transição dos cuidados.

Entre os fatores de risco para eventos cardíacos agudos, o uso de drogas ilícitas está bem estabelecido. A maior preocupação sobre o uso 
de drogas ilícitas após a cirurgia está associada à alta mortalidade em longo prazo e ao número de reinfecções valvares. Devido a isso, recomenda-se a participação em programas de reabilitação após a alta hospitalar ${ }^{(44-45)}$. Observa-se que são escassos os estudos sobre o uso de drogas ilícitas no pós-operatório de cirurgias cardíacas. Além disso, as diretrizes também não incluíram essa abordagem.

As orientações a respeito de hipertermia e febre justificam-se, devido às complicações infecciosas, comuns no pós-operatório de cirurgias cardíacas. Sabe-se que as infecções hospitalares, especialmente de sítio cirúrgico, prolongam o período de internação, geram readmissão e aumentam os custos do tratamento ${ }^{(46-47)}$. Ensinar o paciente e seus familiares a identificar os sinais e sintomas mais comuns de complicações pós-operatórias permite o diagnóstico e a intervenção precoces e, por isso, deve compor as orientações para a alta hospitalar.

As diretrizes defendem que deve ser individualizado o processo de alta hospitalar e incentivada a participação ativa dos pacientes nos processos decisórios, para que eles entendam os fatores que podem ser modificados, compreendam a importância da adesão ao tratamento e do seu envolvimento no processo de reabilitação cardíaca. Essas orientações devem ser ofertadas pelos profissionais de saúde de forma clara, com uma linguagem objetiva para o esclarecimento de dúvidas e incertezas, deixando os pacientes cientes sobre o seu papel para a melhora da qualidade de vida e assim, efetivamente, visualizarem a tão esperada melhora clínica ${ }^{(20-22)}$.

Uma limitação deste estudo inclui a carência de estudos atuais sobre as orientações específicas voltadas ao pós-operatório de cirurgias de correção valvar. Outras limitações são a data de publicação das diretrizes brasileiras mais recentes, bem como a escassez de capítulos que contemplem a abrangência necessária de temas para compor orientações para o autocuidado após a alta hospitalar desse grupo de pacientes.

\section{Conclusão}

As orientações de saúde identificadas para o desempenho do autocuidado após alta hospitalar, no pós-operatório de cirurgia cardíaca, envolvem: atividades de vida diária, alimentação, atividade e exercício físico, sintomas psicológicos, controle de fatores de risco, complicações, terapia medicamentosa, manejo dos sintomas e cuidados com a pele.

A maioria dessas orientações foi mencionada em, no mínimo, dois artigos da revisão. Dentre as mais citadas estão a administração de medicamentos, as reações emocionais, a constipação e a dor. Atividades sociais, depressão, uso de drogas ilícitas, hipertermia, febre, aquisição e interação de medicamentos foram orientações pouco citadas, apesar de sua importância para a reabilitação, no impacto da mortalidade, na identificação precoce de sinais de complicações e para a adesão medicamentosa.

O levantamento dos principais temas para orientação efetiva facilita a criação e implementação de tecnologias educacionais capazes de empoderar o paciente em pós-operatório de cirurgia cardíaca a incorporar ações para o autocuidado.

\section{Colaborações:}

1 - concepção, projeto, análise e interpretação dos dados: Thaina Laurentino Santos, Manoela Gomes Grossi Laprano e Ana Paula da Conceição;

2 - redação do artigo e revisão crítica relevante do conteúdo intelectual: Thaina Laurentino Santos e Manoela Gomes Grossi Laprano;

3 - aprovação final da versão a ser publicada: Manoela Gomes Grossi Laprano e Ana Paula da Conceição.

\section{Referências}

1. Organização Mundial da Saúde. Doenças cardiovasculares [Internet]. Brasília (DF); 2017 [cited 
2019 Jan 8]. Available from: https://www.paho.org/ bra/index.php?option=com_content\&view=article \&id=5253:doencas-cardiovasculares\&Itemid=1096

2. Brasil. Ministério da Saúde. Informações de Saúde. DATASUS Tecnologia da Informação a Serviço do SUS [Internet]. Brasília (DF); 2015 [cited 2015 Sep 5]. Available from: http://datasus.saude.gov.br/

3. Dordetto PR, Pinto GC, Rosa TCSC. Pacientes submetidos à cirurgia cardíaca: caracterização sociodemográfica, perfil clínico-epidemiológico e complicações. Rev Fac Ciênc Méd Sorocaba. 2016;18(3):144-9. DOI: 10.5327/Z1984-4840201625868

4. Rushton M, Howarth M, Grant MJ, Astin F. Personcentred discharge education following coronary artery bypass graft: A critical review. J Clin Nurs. 2017 Dec;26(23-24):5206-15. DOI: 10.1111/ jocn. 14071

5. Contrin LM, Beccaria LM, Werneck AL, Rodrigues AMS, Castro GT, Teixeira CV. Complicações pós-operatórias cardiocirúrgicas e tempo de internação. Rev enferm UFPE. 2018 ago; 12(8):2105-12. DOI: https://doi.org/10.5205/19818963-v12i8a234846p2105-2112-2018

6. Weber LAF, Lima MADS, Acosta AM, Marques GQ. Transição do cuidado do hospital para o domicílio: revisão integrativa. Cogitare Enferm. 2017;22(3):e47615. DOI: http://dx.doi. org/10.5380/ce.v22i3.47615

7. Acosta AM, Câmara CE, Weber LAF, Fontenele RM. Atividades do enfermeiro na transição do cuidado: realidades e desafios. Rev enferm UFPE on line. 2018 dez;12(12):3190-7. DOI: https://doi.org/10.5205/1981-8963v12i12a231432p3190-3197-2018

8. Neves ACOJ, Castro EAB, Costa SRD. Necessidades de cuidados domiciliares de enfermagem após a alta hospitalar no contexto do SUS. Cogitare Enferm. 2016 out/dez;21(4):1-10. DOI: http:// dx.doi.org/10.5380/ce.v21i4.47708

9. NunesHJM,QueirósPJP.Patientwithstroke:hospital discharge planning, functionality and quality of life. Rev Bras Enferm. 2017;70(2):415-23. DOI: http://dx.doi.org/10.1590/0034-7167-2016-0166

10. Moura RS, Saraiva FJC, Santos RM, Rosa RFN, Rocha KRSL, Cavalcanti RC. Experiências de pacientes após as cirurgias de revascularização miocárdica: histórias de vida. Rev enferm UFPE on line. 2017 dez;11(Supl. 12):5221-30. DOI: https://doi.org/10.5205/1981-8963v11i12a22863p5221-5230-2017
11. Silva Silva V, Espinoza Quiroz P, Weiss M. Perceptions in Discharge Preparation in MedicalSurgical Patients of a High Complexity Hospital. Index Enferm [Internet]. 2018 ene-jun [cited 2019 Feb 25];27(1/2):23-7. Available from: http://scielo.isciii.es/scielo.php?pid=S1132$12962018000100005 \&$ script=sci_abstract\&tlng $=$ en

12. Weerahandi H, Ziaeian B, Fogerty RL, Jenq GY, Horwitz LI. Predictors for Patients Understanding Reason for Hospitalization. PLoS One. 2018;13(4):e0196479. DOI: https://doi.org/10.1371/ journal.pone.019647

13. Azevedo AMGB, Silva DO, Gomes LOS. Educação em saúde como ferramenta no conhecimento do usuário com hipertensão arterial. Rev enferm UFPE on line. 2017;11(supl. 8):3279-89. DOI: 10.5205/ reuol.11135-99435-1-ED.1108sup201712

14. Riegel B, Dickson VV, Faulkner KM. The Situation-Specific Theory of Heart Failure SelfCare: Revised and Updated. J Cardiovasc Nurs. 2016 May-Jun;31(3):226-35. DOI: 10.1097/JCN. 0000000000000244

15. Lee KS, Moser DK, Pelter MM, Nesbitt T, Dracup K. Autocuidado em residentes rurais com insuficiência cardíaca: o que estamos perdendo. Rev Eur Enferm Cardiovasc. 2016;16(4). DOI: https://doi.org/10.1177/1474515116666439

16. Odom-Forren J, Wesmiller S. Managing Symptoms: Enhancing Patients Self-Management Knowledge and Skills for Surgical Recovery. Semin Oncol Nurs. 2017 Feb;33(1):52-60. DOI: https://doi. org/10.1016/j.soncn.2016.11.005

17. Ramos CFV, Araruna RC, Lima CMF, Santana CLA, Tanaka LH. Education practices: research-action with nurses of Family Health Strategy. Rev Bras Enferm [Internet]. 2018;71(3):1144-51. DOI: http:// dx.doi.org/10.1590/0034-7167-2017-0284

18. Cieto BB, Garbuio DC, Camargo VB, Napoleão AA. Recursos e inovações de enfermagem para a alta: revisão integrativa. Rev Min Enferm. 2014 jul/ set;18(3):752-7. DOI: 10.5935/1415-2762.20140055

19. Inácio LA, Montezeli JH, Sade PMC, Caveião C, Hey AP. Atuação do enfermeiro nas orientações de alta ao paciente pós-transplante renal. Rev Enferm UFSM. 2014 abr/jun;4(2):323-31. DOI: $10.5902 / 2179769210186$

20. Neumann F-J, Sousa-Uva M, Ahlsson A, Alfonso F, Banning AP, Benedetto U, et al. 2018 ESC/EACTS Guidelines on Myocardial Revascularization. The Task Force on Myocardial Revascularization 
of the European Society of Cardiology (ESC) and European Association for Cardio-Thoracic Surgery (EACTS). European Heart J. 2019 Jul-Aug; 20(7-8 Suppl 1):1S-61S. DOI: 10.1714 / 3203.31801

21. Tarasoutchi F, Montera MW, Grinberg M, Barbosa MR, Piñeiro DJ, Sánchez CRM, et al. Diretriz Brasileira de Valvopatias - SBC 2011 / I Diretriz Interamericana de Valvopatias - SIAC 2011. Arq Bras Cardiol [Internet]. 2011 [cited 2019 Feb 15];97(5 supl.1):1-67. Available from: http://publicacoes.cardiol.br/consenso/2011/ Diretriz\%20Valvopatias\%20-\%202011.pdf

22. Nicolau JC, Timerman A, Marin-Neto JA, Piegas LS, Barbosa CJDG, Franci A, et al. Diretrizes da Sociedade Brasileira de Cardiologia sobre Angina Instável e Infarto Agudo do Miocárdio sem Supradesnível do Segmento ST (II Edição, 2007) - Atualização 2013/2014. Arq Bras Cardiol [Internet]. 2014 mar [cited 2019 Feb 10];102(3 Supl.1):1-61. Available from: http://publicacoes. cardiol.br/consenso/2014/Diretriz_de_IAM.pdf

23. Whittemore R, Knafl K. The integrative review: updated methodology. J Adv Nurs. 2005 Dec;52(5):546-53. DOI: 10.1111 / j.13652648.2005.03621.x

24. Ursi ES. Prevenção de lesões de pele no perioperatório: revisão integrativa da literatura [dissertação]. Ribeirão Preto: Escola de Enfermagem de Ribeirão Preto, Universidade de São Paulo; 2005 [cited 2019 Jan 30]. Available from: https:// doi.org/10.1590/S0104-11692006000100017

25. Melnyk BM, Fineout-Overholt E. Making the case for evidence-based practice. In: Melnyk BM, Fineout-Overholt E, editors. Evidence-Based Practice in Nursing \& Healthcare. A Guide to Best Practice. Philadelphia: Lippincott Williams \& Wilkins; 2011. p. 3-24.

26. Yildiz T, Gürkan S, Gür Ö, Ünsal C, Göktaş SB, Özen Y. Effect of Standard Versus Patient-Targeted In-Patient Education on Patients' Anxiety About Self-Care After Discharge From Cardiovascular Surgery Clinics. Cardiovasc J Afr. 2014;25(6):259-64. DOI:10.5830/CVJA-2014-04

27. Fredericks S. The Influence of Country of Origin on Engagement in Self-Care Behaviours Following Heart Surgery: A Descriptive Correlational Study. J Clin Nurs. 2012 Aug;21(15-16):2202-8. DOI: 10.1111/j.1365-2702.2012.04095.x

28. Fredericks S. Timing for Delivering Individualized Patient Education Intervention to Coronary Artery
Bypass Graft Patients: An RCT. Eur J Cardiovasc Nurs. 2009 Jun;8(2):144-50. DOI: 10.1016/j. ejcnurse.2008.10.007

29. Cebeci $\mathrm{F}_{s}$ Celik SS. Discharge training and counselling increase self-care ability and reduce postdischarge problems in CABG patients. J Clin Nurs. 2008 Feb;17(3):412-20. DOI: 10.1111/ j.1365-2702.2007.01952.x

30. Fredericks S, Sidani S, Shugurensky D. The effect of anxiety on learning outcomes post-CABG. Can J Nurs Res [Internet]. 2008 Mar [cited 2019 Jan 22];40(1)127-40. Available from: https://www. researchgate.net/publication/5394262_The_effect_ of_anxiety_on_learning_outcomes_post-CABG

31. Gentil LLS, Silva RM, Benavente SBT, Costa ALS. Manual educativo de cuidados no pós-operatório de revascularização miocárdica: uma ferramenta para pacientes e familiares. Rev Eletr Enf. 2017;19:a38. DOI: http://dx.doi.org/10.5216/ree. v19.43068

32. Guzinski C, Lopes ANM, Flor J, Migliavaca J, Tortato C, Dal Pai D. Boas práticas para comunicação efetiva: a experiência do round interdisciplinar em cirurgia ortopédica. Rev Gaúcha Enferm. 2019;40(esp):e20180353. DOI: https://doi.org/10.1590/1983- 1447.2019.20180353

33. Sobral PD, Oliveira DC, Gomes ET, Carvalho PO, Brito NMT, Oliveira DAGC, et al. Razões para não adesão a fármacos em pacientes com doença arterial coronariana. Rev Soc Bras Clin Med[Internet]. 2017 jul-set [cited 2019 Feb 15];15(3):166-70. Available from: https:// https://pesquisa.bvsalud. org/ripsa/resource/pt/biblio-875437

34. Moreira MB, Mesquita MGR, Stipp MAC, Paes GO. Potential intravenous drug interactions in intensive care. Rev Esc Enferm USP. 2017;51:e03233. DOI: http://dx.doi.org/10.1590/ S1980-220X2016034803233

35. Açıkel MET. Evaluation of Depression and Anxiety in Coronary Artery Bypass Surgery Patients: A Prospective Clinical Study. Braz J Cardiovasc Surg. 2019;34(4):389-95. DOI: 10.21470/1678-9741-2018-0426

36. Ribeiro CP, Silveira CO, Benetti ERR, Gomes JS, Stumm EMF. Diagnósticos de enfermagem em pacientes no pós-operatório de cirurgia cardíaca. Rev RENE. 2015 mar-abr;16(2):159-67. DOI: 10.15253/2175-6783.2015000200004

37. Menezes TC, Bassi D, Cavalcanti RC, Barros JESL, Granja KSB, Calles ACN, et al. Comparações 
e correlações da intensidade da dor e da força muscular periférica e respiratória no pré e pós-operatório de cirurgia cardíaca. Rev bras ter intensiva. 2018 Oct/Dec;30(4):479-86. DOI: http:// dx.doi.org/10.5935/0103-507x.20180069

38. Dessotte CAM, Rodrigues HF, Furuya RK, Rossi LA, Dantas RAS. Stressors perceived by patients in the immediate postoperative of cardiac surgery. Rev Bras Enferm. 2016;69(4):694-703. DOI: http://dx.doi.org/10.1590/0034-7167.2016690418i

39. Chiapinotto S, Dallazen F, Bodnar ET, Winkelman ER. Nívelde dor egrau de independência funcional de pacientes submetidos à cirurgia cardíaca. Rev baiana enferm. 2017;31(4):e21388. DOI: http://dx.doi.org/10.18471/rbe.v31i4.21388

40. Ribeiro KRA, Gonçalves FAF, Borges MM, Loreto RGO, Amaral MS. Pós-Operatório de Revascularização do Miocárdio: Possíveis Diagnósticos e Intervenções de Enfermagem. J res: fundam Care Online. 2019 abr/jun;11(3):801-8. DOI: http:// dx.doi.org/10.9789/2175-5361.2019. v11i3.801-808

41. Orem DE. Nursing: concepts of practice. 6th ed. St Louis (USA): Mosby Inc; 2001.

42. Alves MP, Lanzoni GMM, Koerich C, Higashi GDC, Baggio MA, Erdmann A. O processo de viver a cirurgia de revascularização cardíaca: uma análise de gênero. Esc Anna Nery [online]. 2016;20(4):e20160093. DOI: https://doi. org/10.5935/1414-8145.20160093
43. Leigh-Hunt N, Bagguley D, Bash K, Turner V, Turnbull S, Valtorta N, et al. An Overview of Systematic Reviews on the Public Health Consequences of Social Isolation and Loneliness. Public Health. 2017 Nov;152:157-71. DOI: 10.1016/j.puhe.2017.07.035

44. Osterdal OB, Salminen P-R, Jordal S, Sjursen H, Wendelbo O, Haaverstad R. Cardiac Surgery for Infective Endocarditis in Patients With Intravenous Drug Use. Interact Cardiovasc Thorac Surg. 2016 May;22(5):633-40. DOI: 10.1093/icvts/ivv397

45. van Wijk XMR, Vittinghoff E, Wu AHB, Lynch KL, Riley ED. Cocaine Use Is Associated With a Higher Prevalence of Elevated ST2 Concentrations. Clin Biochem. 2017 Sep;50(13-14):791-3 DOI: 10.1016/j. clinbiochem.2017.04.015

46. Beccaria LM, Cesarino CB, Werneck AL, Góes NC, Santos KS, Machado MN. Complicações pós-operatórias em pacientes submetidos à cirurgia cardíaca em hospital de ensino. Arq Ciênc Saúde. 2015 jul-set;22(3):37-41. DOI: 10.17696/2318-3691.22.3.2015.216

47. Braz NJ, Evangelista SS, Evangelista SS, Garbaccio JL, Oliveira AC. Infecção do sítio cirúrgico em pacientes submetidos a cirurgias cardíacas: uma análise do perfil epidemiológico. Rev Enferm Centro-Oeste Mineiro. 2018;8:e1793. DOI: https://doi.org/10.19175/recom.v8i0.1793

Recebido: 30 de janeiro de 2020

Aprovado: 7 de maio de 2020

Publicado: 27 de julho de 2020

A Revista Baiana de Enfermagem utiliza a Licença Creative Commons - Atribuição-NãoComercial 4.0 Internacional. https://creativecommons.org/licenses/by-nc/4.0/

Este artigo é de acesso aberto distribuído sob os termos da Licença Creative Commons (CC BY-NC). Esta licença permite que outros remixem, adaptem e criem a partir do seu trabalho para fins não comerciais. Embora os novos trabalhos tenham de lhe atribuir o devido crédito e não possam ser usados para fins comerciais, os usuários não têm de licenciar esses trabalhos derivados sob os mesmos termos. 